\title{
Zero Eigenvalue Analysis for the Determination of Multiple Steady States in Reaction Networks
}

\author{
Hsing-Ya Li \\ Department of Chemical Engineering, National Lien Ho College of Technology and Commerce, \\ Miaoli, Taiwan 360, R.O.C.
}

Z. Naturforsch. 53a, 171-177 (1998); received December 31, 1997

\begin{abstract}
A chemical reaction network can admit multiple positive steady states if and only if there exists a positive steady state having a zero eigenvalue with its eigenvector in the stoichiometric subspace. A zero eigenvalue analysis is proposed which provides a necessary and sufficient condition to determine the possibility of the existence of such a steady state. The condition forms a system of inequalities and equations. If a set of solutions for the system is found, then the network under study is able to admit multiple positive steady states for some positive rate constants. Otherwise, the network can exhibit at most one steady state, no matter what positive rate constants the system might have. The construction of a zero-eigenvalue positive steady state and a set of positive rate constants is also presented. The analysis is demonstrated by two examples.
\end{abstract}

Key words: Reaction Network; Multiple Steady States; Zero Eigenvalue Analysis.

\section{Introduction}

It is well known that certain nonlinear models of chemical systems can exhibit several positive steady states for some particular system parameters. (A positive steady state is one for which all species have positive concentrations.) On the other hand, there are many chemical systems which can admit at most one positive steady state, no matter what positive rate constants the systems might have. The development of methods to distinguish these two types of systems is the aim of this article. Such identifications not only help engineers to design more efficient and safer reaction processes but also help chemists to explain experimental results.

In this field, as in any other part of reaction kinetics, there are two approaches [1]: investigation of simple but possibly realistic models or a search for general criteria that ensure or exclude multistationarity in large classes of mechanisms. Some examples for this approach are given by Othmer [2], Luss [3, 4], Gray and Scott [5, 6]. The second approach was initiated by Horn, Jackson and Feinberg. Their results are based upon classification of reaction networks by means of a non-negative integer index called the deficiency, which is determined by the reaction network structure [7]. Work of Horn [8], Horn and Jackson [9], and Feinberg [10,11] led to the deficiency

Reprint requests to Prof. H.-Y. Li; Fax: (37) 357301 ;

E-mail: hyli@mail.lctc.edu.tw zero theorem. Feinberg developed the deficiency one theorem [7] and the deficiency one algorithm [12]. The stoichiometric network analysis by Clarke [13] determines the stability of chemical networks, which also provides some information about steady-state multiplicities. Also Willamowski [14] contributed to the theory of mass action kinetics.

In this work we show that a network has the capacity to admit multiple positive steady states if and only if it contains a positive steady state having a zero eigenvalue with its corresponding eigenvector lying in its stoichiometric subspace. Then a zero eigenvalue analysis is proposed to determine the possibility of the existence of such a steady state in a reaction network. The assumptions are: the systems are isothermal, the reactions are mass actions, and the steady states are positive. The construction of a zero-eigenvalue positive steady state and a set of corresponding positive rate constants is also proposed. The method is demonstrated by two examples.

\section{Theoretical Background}

\subsection{Some Terminology}

Our introduction of terminology will be casual, relying more on examples than on formal definitions. (Formal definitions can be found in [11].) Consider the FieldNoyes [15] "Oregonator" model for the Belousov-Zha- 
botinskii system:

$$
\begin{aligned}
& A_{2} \rightarrow A_{1} \rightarrow 2 A_{1}+A_{3}, \\
& A_{1}+A_{2} \rightarrow 0 \leftarrow 2 A_{1}, \\
& A_{3} \rightarrow f A_{2} .
\end{aligned}
$$

(The Field-Noyes "stoichiometric parameter" $f$ is a positive real number.) The zero complex " 0 " comes from the assumption that the concentrations of the products produced by the reactants $A_{1}$ and $A_{2}$ as well as by $2 A_{1}$ remain constant during the reaction period.

We will use the symbol $N$ to denote the number of species in a network under consideration. Thus, for network (1) $N=3$. By $\Re^{N}$ we shall mean the usual vector space of $N$-tuples of real numbers. The standard basis for $\Re^{N}$ will be denoted $\left\{\boldsymbol{A}_{1}, \boldsymbol{A}_{2}, \ldots, \boldsymbol{A}_{N}\right\}$.

The complexes of a network are the objects that appear before and after reaction arrows. Thus the set of complexes for network (1) is $\left\{A_{2}, A_{1}, 2 A_{1}+A_{3}, A_{1}+A_{2}, 0\right.$, $\left.2 A_{1}, A_{3}, f A_{2}\right\}$. Given a network with $N$ species, we shall associated with each complex a vector in $\Re^{N}$. Consider the network (1). With the complex $A_{1}$ we associate the complex vector $A_{1}$ in $\Re^{3}$; with the complex $A_{1}+A_{2}$ we associate the complex vector $\boldsymbol{A}_{1}+\boldsymbol{A}_{2}$; and so on.

We write $y_{i} \rightarrow y_{j}$ (or the abbreviation $i \rightarrow j$ ) to indicate the reaction whereby the complex $y_{i}$ reacts to complex $y_{j}$. The symbol $R$ denotes the set of reactions in a network. Thus the set of reactions in network (1) is, $R=\left\{A_{2} \rightarrow A_{1}, A_{1} \rightarrow 2 A_{1}+A_{3}, A_{1}+A_{2} \rightarrow 0,2 A_{1} \rightarrow 0\right.$, $\left.A_{3} \rightarrow f A_{2}\right\}$. The symbol $r$ is reserved for the number of distinct reactions in a network. For network (1), $r=5$. A reaction $y_{i} \rightarrow y_{j}$ is said to be reversible if its reverse reaction $y_{j} \rightarrow y_{i}$ is also in the network; otherwise, the reaction $y_{i} \rightarrow y_{j}$ is irreversible. We shall call $y_{i} \rightleftarrows y_{j}$ a reversible reaction pair. The symbol $p$ denotes the number of distinct reversible reaction pairs in a network. The number of irreversible reactions in a network is $r-2 p$. For network (1), there are five irreversible reactions without $(p=0)$ any reversible reaction pair.

We associate each reaction $y_{i} \rightarrow y_{j}$ a reaction vector, $\boldsymbol{y}_{j}-\boldsymbol{y}_{i} \in \Re^{N}$, obtained by subtracting the "reactant" complex vector $\boldsymbol{y}_{i}$ from the "product" complex vector $\boldsymbol{y}_{j}$. Consider network (1). For the reaction $A_{1}+A_{2} \rightarrow 0$, the corresponding reaction vector in $\Re^{3}$ is $0-\left(\boldsymbol{A}_{1}+\boldsymbol{A}_{2}\right)=-\boldsymbol{A}_{1}-\boldsymbol{A}_{2}$. The set of reaction vectors of network (1) is

$$
\left\{A_{1}-A_{2}, A_{1}+A_{3},-A_{1}-A_{2},-2 A_{1}, f A_{2}-A_{3}\right\} \text {. }
$$

A reaction network has rank $s$ if there exists a linearly independent set of $s$ reaction vectors for the network and there exists no linearly independent set of $s+1$ reac- tion vectors. Consider network (1). The set of three reaction vectors $\left\{\boldsymbol{A}_{1}-\boldsymbol{A}_{2}, \boldsymbol{A}_{1}+\boldsymbol{A}_{3},-\boldsymbol{A}_{1}-\boldsymbol{A}_{2}\right\}$ is linearly independent, but any set of four reaction vectors for network (1) is linearly dependent. Thus, the rank of network (1) is three, and for it we write $s=3$.

The stoichiometric subspace for a network is the span of its reaction vectors. The symbol $S_{t}$ designates the stoichiometric subspace for a network. It is clear that a stoichiometric subspace is a linear subspace of $\Re^{N}$. The dimension of a stoichiometric subspace is equal to the rank $s$ of its network. The stoichiometric subspace for network (1) is the span generated by the reaction vectors given in (2). It is identical to $\Re^{3}$.

A vector $\phi \in \Re^{N}$ is sign-compatible with $S_{t}$ if there exists in $S_{t}$ a vector $\sigma \in \Re^{N}$ such that the sign $\sigma_{L}=\operatorname{sign} \phi_{L}, L=1,2, \ldots, N$. This means that $\sigma_{L}$ is positive if $\phi_{L}$ is positive, $\sigma_{L}$ is negative if $\phi_{L}$ is negative, and $\sigma_{L}$ is zero if $\phi_{L}$ is zero. This requirement comes from the mass conservation condition of a system. Since the stoichiometric subspace for network (1) is $\Re^{3}$, any vector in $\Re^{3}$ is sign compatible with $S_{t}$ for network (1).

Consider a network with $r$ distinct reactions, $p$ reversible reaction pairs, and $r-2 p$ irreversible reactions. A spanning subnetwork of a network under consideration consists of all the $r-2 p$ irreversible reactions and one (and only one) reaction of each $p$ reversible reaction pair. There are $r-p$ reactions in a spanning subnetwork. The symbol $F$ is used to denote the set of reactions in a spanning subnetwork. Since all the reactions in network (1) are irreversible, the spanning subnetwork for network (1) is exactly itself and the set $F$ is identical to the reaction set $R$.

There are more than one spanning subnetworks for a network having one or more than one reversible reaction pairs $(p \geq 1)$. In each case under study, we choose to work with a fixed (but arbitrary) spanning subnetwork. For a chosen spanning subnetwork, we shall construct a set of corresponding spanning-subnetwork vectors $\left\{d^{(1)}, d^{(2)}\right.$, $\left.\ldots, \boldsymbol{d}^{(r-p-s)}\right\}$ in $\Re^{R}$. Let $\left\{\boldsymbol{\omega}_{i \rightarrow j}: i \rightarrow j \in R\right\}$ be the standard basis for $\Re^{R}$. These spanning-subnetwork vectors are the $r-p-s$ linearly independent (nonzero) solutions of the vector equation

$$
\sum_{i \rightarrow j \in F} d_{i \rightarrow j}^{(L)}\left(\boldsymbol{y}_{j}-\boldsymbol{y}_{i}\right)=\mathbf{0}, \quad L=1,2, \ldots, r-p-s
$$

(A solution is a family of number $\left\{d_{i \rightarrow j}^{(L)}: i \rightarrow j \in F\right\}$ ). Then $d^{(L)}, L=1,2, \ldots, r-p-s$, are vectors defined in the following way:

$$
\boldsymbol{d}^{(L)}=\sum_{i \rightarrow j \in F} d_{i \rightarrow j}^{(L)} \boldsymbol{\omega}_{i \rightarrow j} .
$$


Computing the spanning-subnetwork vectors for network (1), by (3a) $\boldsymbol{d}^{(1)}, \boldsymbol{d}^{(2)}(r-p-s=5-0-3)$ satisfy

$$
\begin{aligned}
& d_{A_{2} \rightarrow A_{1}}^{(L)}\left(\boldsymbol{A}_{1}-\boldsymbol{A}_{2}\right) \\
& +d_{A_{1} \rightarrow 2 A_{1}+A_{3}}^{(L)}\left(2 \boldsymbol{A}_{1}+\boldsymbol{A}_{3}-\boldsymbol{A}_{1}\right) \\
& +d_{A_{1}+A_{2} \rightarrow 0}^{(L)}\left(\mathbf{0}-\boldsymbol{A}_{1}-\boldsymbol{A}_{2}\right) \\
& +d_{2 A_{1} \rightarrow 0}^{(L)}\left(\mathbf{0}-2 \boldsymbol{A}_{1}\right) \\
& +d_{A_{3} \rightarrow f A_{2}}^{(L)}\left(f \boldsymbol{A}_{2}-\boldsymbol{A}_{3}\right)=\mathbf{0}, L=1,2 .
\end{aligned}
$$

Two nonzero linearly independent solutions to the above equation are

$$
\begin{aligned}
& d_{A_{2} \rightarrow A_{1}}^{(1)}=-1, \quad d_{A_{1} \rightarrow 2 A_{1}+A_{3}}^{(1)}=0, \\
& d_{A_{1}+A_{2} \rightarrow 0}^{(1)}=1, \quad d_{2 A_{1} \rightarrow 0}^{(1)}=-1, \\
& d_{A_{3} \rightarrow f A_{2}}^{(1)}=0, \\
& d_{A_{2} \rightarrow A_{1}}^{(2)}=0, \quad d_{A_{1} \rightarrow 2 A_{1}+A_{3}}^{(2)}=-1, \\
& d_{A_{1}+A_{2} \rightarrow 0}^{(2)}=-f, \quad d_{2 A_{1} \rightarrow 0}^{(2)}=\frac{f-1}{2}, \\
& d_{A_{3} \rightarrow f A_{2}}^{(2)}=-1 .
\end{aligned}
$$

Consider a reaction network with $N$ species $A_{1}, A_{2}$, $\ldots, A_{N}$. Let $c=\left[c_{1}, c_{2}, \ldots, c_{N}\right]$ be a composition vector in $\overline{\mathrm{P}}^{N}$ (non-negative orthant of $\Re^{N}$ ) for species $A_{L}$. $L=1,2, \ldots, N$. In general, the set of isothermal mass action differential equations describing the behavior of a reaction network can be written

$$
\begin{aligned}
\frac{\mathrm{d} \boldsymbol{c}}{\mathrm{d} t} & =\boldsymbol{f}(\boldsymbol{c})=\left(f_{1}(\boldsymbol{c}), \ldots, f_{N}(\boldsymbol{c})\right) \\
& =\sum_{i \rightarrow j \in R} k_{i \rightarrow j}\left(\prod_{L=1}^{N} c_{L}^{y_{i L}}\right)\left(\boldsymbol{y}_{j}-\boldsymbol{y}_{i}\right),
\end{aligned}
$$

$\boldsymbol{y}_{i}$ and $\boldsymbol{y}_{j}$ denoting respectively the reactant and product complex vectors, $y_{i L}$ denoting the stoichiometric coefficient of species $A_{L}$ in the reactant complex $y_{i}$, and $k_{i \rightarrow j}$ denoting the rate constant for the reaction $i \rightarrow j$. By a positive steady state of a reaction system we shall mean a composition $c^{*} \in \mathrm{P}^{N}$ (positive orthant of $\Re^{N}$ ) such that $\boldsymbol{f}\left(\boldsymbol{c}^{*}\right)=\mathbf{0}$. The mass action differential equations for network (1) are

$$
\begin{aligned}
\frac{\mathrm{d} c_{1}}{\mathrm{~d} t}= & k_{A_{2} \rightarrow A_{1}} c_{2}+k_{A_{1} \rightarrow 2 A_{1}+A_{3}} c_{1} \\
& -k_{A_{1}+A_{2} \rightarrow 0} c_{1} c_{2}-2 k_{2 A_{1} \rightarrow 0} c_{1}^{2}, \\
\frac{\mathrm{d} c_{2}}{\mathrm{~d} t}= & -k_{A_{2} \rightarrow A_{1}} c_{2}-k_{A_{1}+A_{2} \rightarrow 0} c_{1} c_{2} \\
& +f k_{A_{3} \rightarrow f A_{2}} c_{3}, \\
\frac{\mathrm{d} c_{3}}{\mathrm{~d} t}= & k_{A_{1} \rightarrow 2 A_{1}+A_{3}} c_{1}-k_{A_{3} \rightarrow f A_{2}} c_{3} .
\end{aligned}
$$

The right hand side of $(5 \mathrm{a})$ is a linear combination of all reaction vectors in its network for any composition $\boldsymbol{c} \in \overline{\mathbf{P}}^{N}$. This indicates that $\mathrm{d} c / \mathrm{d} t$ always lies in the stoichiometric subspace $S_{t}$ of the network under consideration. Moreover, a composition $c_{1}$ can evolve to a composition $c_{2}$ only if such a composition change $c_{2}-c_{1}$ lies in $S_{t}$. Motivated by these considerations, we say that two compositions $c_{1}$ and $c_{2}$ are stoichiometrically compatible if $c_{2}-c_{1} \in S_{t}$. Thus, as a network is said to have the capacity to admit multiple positive steady states, it really means that there exists a set of positive rate constants such that its corresponding isothermal mass action differential equation (5a) admits at least two stoichiometrically compatible positive steady states. Since the stoichiometric subspace for network (1) is $\Re^{3}$, any two positive steady states are stoichiometrically compatible.

\section{Results and Discussion}

\subsection{A Necessary and Sufficient Condition}

Based on an idea of Feinberg, Li [16] constructed a necessary and sufficient condition to determine the possibility of multiple positive steady states in forest-like networks, whose structures contain no loops. This condition is then extended to cover general mass action networks which may or may not be forest-like [17]. It has been shown [17] that if a network has the capacity to admit multiple positive steady states, then it admits two positive steady states for which the distance between them tends to zero. It is obvious that, if a network admits two positive steady states, whether the distance between them approaches zero or not, it exhibits multiple steady states. Let $c^{*}$ and $c^{* *}$ be two steady states for (5a). We have

$$
\begin{aligned}
& \boldsymbol{f}\left(\boldsymbol{c}^{*}\right)=\mathbf{0}, \\
& \boldsymbol{f}\left(\boldsymbol{c}^{* *}\right)=\mathbf{0} .
\end{aligned}
$$

Since $c^{*}-c^{* *} \in S_{t}$, we can write $c^{*}=c^{* *}+\lambda \gamma$, where $\lambda$ is a real number and $\gamma$ is a vector in $S_{t}$. Equation (6) indicates that

$$
\frac{f\left(c^{* *}\right)-\boldsymbol{f}\left(c^{*}\right)}{\lambda}=\mathbf{0} .
$$

As $c^{* *}$ approaches $c^{*}, \lambda$ tends to zero. Equation ( $\left.7 \mathrm{a}\right)$ becomes

$$
\begin{aligned}
& \lim _{\lambda \rightarrow 0} \frac{f\left(c^{* *}\right)-f\left(c^{*}\right)}{\lambda} \\
& =\lim _{\lambda \rightarrow 0} \frac{f\left(c^{*}+\lambda \gamma\right)-f\left(c^{*}\right)}{\lambda}=F\left(c^{*}\right) \gamma=\mathbf{0},
\end{aligned}
$$


where $\boldsymbol{F}\left(c^{*}\right) \boldsymbol{\gamma}$ is the derivative of the rate function $\boldsymbol{f}(\boldsymbol{c})$ in $(5 \mathrm{a})$, evaluated at steady state $c^{*}$ along the direction $\gamma$. The combination of (6a) and (7b) forms a necessary and sufficient condition for multiple positive steady states. It says that a network can exhibit steady state multiplicity if and only if there exists a positive steady state $c^{*}$, (6a), having a zero eigenvalue, (7b), with its corresponding eigenvector $\gamma \in S_{t}$.

\subsection{The Zero Eigenvalue Analysis}

For a given reaction network, the zero eigenvalue analysis proposed below is used to determinate the possibility that there exists a positive steady state having a zero eigenvalue with its corresponding eigenvector in the stoichiometric subspace. It can be applied to both forest-like and circular networks. For a given reaction network, we choose an arbitrary spanning subnetwork and construct the corresponding spanning-subnetwork vectors. Following the condition proposed below, a system of equations and inequalities is constructed. We then solve the system. If a set of qualified solutions exists, there is a set of positive rate constants such that the corresponding isothermal mass action differential equations for the given network admit a zero-eigenvalue positive steady state. Hence, it has the capacity to admit several positive steady states. Otherwise, no matter what positive rate constants the system might have, the differential equations can exhibit at most one positive steady state.

Zero eigenvalue analysis. Consider an $\mathrm{N}$-species reaction network with a reaction set $R$ and a stoichiometric subspace $S_{t}$. Suppose the network has rank $s$ and $r$ reactions with $p$ reversible reaction pairs. Let the reaction set for an arbitrary spanning subnetwork be $F$ and let $\left\{\boldsymbol{d}^{(1)}, \boldsymbol{d}^{(2)}, \ldots, \boldsymbol{d}^{(r-p-s)}\right\}$ be a set of corresponding spanning-subnetwork vectors. Then the corresponding isothermal mass action differential equations for the given network have the capacity to admit a positive steady state having a zero eigenvalue with its corresponding eigenvector in the stoichiometric subspace $S_{t}$, if and only if there exists a nonzero vector $\phi \in \Re^{N}$ which is sign compatible with $S_{t}$ and also the numbers $\xi_{1}, \xi_{2}, \ldots, \xi_{r-p-s}$, $\alpha_{1}, \alpha_{2}, \ldots, \alpha_{r-p-s}$, satisfying the following two conditions:

(i) For every reversible reaction $y_{i} \rightleftarrows y_{j} \in R$ with $y_{i} \rightarrow y_{j} \in F$,

$$
\sum_{L=1}^{r-p-s}\left[\xi_{L}\left(\boldsymbol{y}_{i} \cdot \boldsymbol{\phi}\right)+\alpha_{L}\right] d_{i \rightarrow j}^{(L)} \text { and }
$$

$$
\sum_{L=1}^{r-p-s}\left[\xi_{L}\left(\boldsymbol{y}_{j} \cdot \boldsymbol{\phi}\right)+\alpha_{L}\right] d_{i \rightarrow j}^{(L)}
$$

are sign compatible with $\left(\boldsymbol{y}_{j}-\boldsymbol{y}_{i}\right) \cdot \boldsymbol{\phi}$.

(ii) For every irreversible reaction $y_{i} \rightarrow y_{j} \in R$,

$$
\begin{aligned}
& \sum_{L=1}^{r-p-s} \xi_{L} d_{i \rightarrow j}^{(L)}>0 \text { and } \\
& \sum_{L=1}^{r-p-s}\left[\xi_{L}\left(\boldsymbol{y}_{i} \cdot \boldsymbol{\phi}\right)+\alpha_{L}\right] d_{i \rightarrow j}^{(L)}=0 .
\end{aligned}
$$

If the answer is yes, the set of solution $\left\{\phi \in \Re^{N}, \xi_{L}\right.$, $\left.\alpha_{L}, L=1, \ldots, r-p-s\right\}$ for the conditions (i) and (ii) can be used to construct a set of positive rate constants $k_{i \rightarrow j}$ and a positive steady state $c^{*}$ having a zero eigenvalue with its corresponding eigenvector $\gamma \in S_{t}$. The steady state $c^{*}$ can be calculated by ( $\left.8 \mathrm{a}\right)$ for any (nonzero) eigenvector $\gamma \in S_{t}$ which is sign compatible with the vector $\phi$ :

$$
\begin{aligned}
c^{*} & =\left[c_{1}^{*}, c_{2}^{*}, \ldots, c_{N}^{*}\right] \\
& =\frac{\gamma}{\boldsymbol{\phi}}=\left[\frac{\gamma_{1}}{\phi_{1}}, \frac{\gamma_{2}}{\phi_{2}}, \ldots, \frac{\gamma_{N}}{\phi_{N}}\right] .
\end{aligned}
$$

The rate constants $\left\{k_{i \rightarrow j}: i \rightarrow j \in R\right\}$ can be computed by (8b), where the variables $\left\{\kappa_{i \rightarrow j}: i \rightarrow j \in R\right\}$ are computed by $(8 \mathrm{c})-(8 \mathrm{~g})$ :

$$
k_{i \rightarrow j}=\frac{\kappa_{i \rightarrow j}}{\prod_{L=1}^{N}\left(c_{L}^{*}\right)^{y_{i L}}} .
$$

For every irreversible reaction $i \rightarrow j \in R$,

$$
\kappa_{i \rightarrow j}=\sum_{L=1}^{r-p-s} \xi_{L} d_{i \rightarrow j}^{(L)} .
$$

For every reversible reaction $i \rightleftarrows j \in R$ with $i \rightarrow j \in F$ and $\boldsymbol{y}_{i} \cdot \boldsymbol{\phi} \neq \boldsymbol{y}_{j} \cdot \boldsymbol{\phi}$

$$
\begin{aligned}
\kappa_{j \rightarrow i}= & \frac{\sum_{L=1}^{r-p-s}\left[\xi_{L}\left(\boldsymbol{y}_{i} \cdot \boldsymbol{\phi}\right)+\alpha_{L}\right] d_{i \rightarrow j}^{(L)}}{\left(\boldsymbol{y}_{j}-\boldsymbol{y}_{i}\right) \cdot \boldsymbol{\phi}}, \\
\kappa_{i \rightarrow j} & =\frac{\sum_{L=1}^{r-p-s}\left[\xi_{L}\left(\boldsymbol{y}_{j} \cdot \boldsymbol{\phi}\right)+\alpha_{L}\right] d_{i \rightarrow j}^{(L)}}{\left(\boldsymbol{y}_{j}-\boldsymbol{y}_{i}\right) \cdot \boldsymbol{\phi}} .
\end{aligned}
$$

For every reversible reaction $i \rightleftarrows j \in R$ with $i \rightarrow j \in F$ and $\boldsymbol{y}_{i} \cdot \boldsymbol{\phi}=\boldsymbol{y}_{j} \cdot \boldsymbol{\phi}$, 


$$
\begin{aligned}
& \kappa_{i \rightarrow j}>0, \quad \kappa_{j \rightarrow i}>0 \\
& \kappa_{i \rightarrow j}-\kappa_{j \rightarrow i}=\sum_{L=1}^{r-p-s} \xi_{L} d_{i \rightarrow j}^{(L)} .
\end{aligned}
$$

\subsection{Applications}

Example 1. Consider the network (1). Substituting the spanning-subnetwork vectors $\boldsymbol{d}^{(1)}, \boldsymbol{d}^{(2)}$ in (4b) and (4c) into condition (ii) of the zero eigenvalue analysis, we have $(9 a)-(9 j)$ :

For an irreversible reaction $A_{2} \rightarrow A_{1}$,

$$
\begin{aligned}
& \xi_{1}(-1)>0, \\
& {\left[\xi_{1}\left(\phi_{2}\right)+\alpha_{1}\right](-1)=0 .}
\end{aligned}
$$

For an irreversible reaction $A_{1} \rightarrow 2 A_{1}+A_{3}$,

$$
\begin{aligned}
& \xi_{2}(-1)>0, \\
& {\left[\xi_{2}\left(\phi_{1}\right)+\alpha_{2}\right](-1)=0 .}
\end{aligned}
$$

For an irreversible reaction $A_{1}+A_{2} \rightarrow 0$,

$$
\begin{aligned}
& \xi_{1}(1)+\xi_{2}(-f)>0, \\
& {\left[\xi_{1}\left(\phi_{1}+\phi_{2}\right)+\alpha_{1}\right](1)} \\
& +\left[\xi_{2}\left(\phi_{1}+\phi_{2}\right)+\alpha_{2}\right](-f)=0 .
\end{aligned}
$$

For an irreversible reaction $2 A_{1} \rightarrow 0$,

$$
\begin{aligned}
& \xi_{1}(-1)+\xi_{2}\left(\frac{f-1}{2}\right)>0, \\
& {\left[\xi_{1}\left(2 \phi_{1}\right)+\alpha_{1}\right](-1)} \\
& +\left[\xi_{2}\left(2 \phi_{1}\right)+\alpha_{2}\right]\left(\frac{f-1}{2}\right)=0 .
\end{aligned}
$$

For an irreversible reaction $A_{3} \rightarrow f A_{2}$,

$$
\begin{aligned}
& \xi_{2}(-1)>0, \\
& {\left[\xi_{2}\left(\phi_{3}\right)+\alpha_{2}\right](-1)=0 .}
\end{aligned}
$$

Equations (9b) and (9d) lead to $\alpha_{1}=-\xi_{1} \phi_{2}$ and $\alpha_{2}=-\xi_{2} \phi_{1}$, respectively. Inserting them into (9f) and (9h), we have

$$
\begin{aligned}
& \xi_{1}=f K \xi_{2}, \\
& (K-2) \xi_{1}+\left(\frac{f-1}{2}\right) \xi_{2}=0,
\end{aligned}
$$

where $K=\phi_{2} / \phi_{1}$. From (10), we obtain

$$
K=1 \pm \frac{\sqrt{2 f^{2}+2 f}}{2 f}
$$

Equations (9c), (9e) and (10a) indicate $K<1$. Since $f$ is a positive real number, the plus term in $(10 \mathrm{c})$ is not satisfied. Substituting (10a) and the minus term of (10c) into (9a) and (9g), we obtain that $f>1$ and $f<1$, respectively. The contradiction says that for any real positive number $f$, there do not exist solutions to the zero eigenvalue analysis. We come to the conclusion that, taken with the mass action kinetics, network (1) cannot admit either a zero-eigenvalue positive steady state or multiple positive steady states, no matter what positive rate constants the system might have.

Example 2. Consider an enzyme-catalyzed reaction [18]:

$$
\begin{aligned}
& \mathrm{S}+\mathrm{E} \rightleftarrows \mathrm{ES} \rightarrow \mathrm{P}+\mathrm{E}, \\
& \mathrm{S}+\mathrm{ES} \rightleftarrows \mathrm{ES}_{2} \rightarrow \mathrm{P}+\mathrm{ES},
\end{aligned}
$$

where $\mathrm{S}$ is a substrate, $\mathrm{E}$ is an enzyme, $\mathrm{ES}$ and $\mathrm{ES}_{2}$ are intermediates and $\mathrm{P}$ is a product. Let the above reactions take place in a well-mixed continuous-flow stirred-tank reactor (CSTR) operated in such a way that $S$ is in the feed stream, $\mathrm{S}$ and $\mathrm{P}$ are in the effluent flow, while E, ES and $\mathrm{ES}_{2}$ are retained in the reactor by using, for example, porous membranes or screens to prevent escape of immobilized enzyme pellets. In reaction network terms [7], the reactions $S \rightleftarrows 0$ are used to describe $S$ in both input and output flows, while the reaction $\mathrm{P} \rightarrow 0$ describes $\mathrm{P}$ in the output flow. Thus, the CSTR system is modeled by the network

$$
\begin{aligned}
& A_{1} \rightleftarrows 0 \leftarrow A_{2}, \\
& A_{1}+A_{3} \rightleftarrows A_{4} \rightarrow A_{2}+A_{3}, \\
& A_{1}+A_{4} \rightleftarrows A_{5} \rightarrow A_{2}+A_{4},
\end{aligned}
$$

where $A_{1}=\mathrm{S}, A_{2}=\mathrm{P}, A_{3}=\mathrm{E}, A_{4}=\mathrm{ES}$ and $A_{5}=\mathrm{ES}_{2}$. Suppose that the temperature, density and volume of the solution do not change substantially with time during operation. According to the law of mass action, this system is describe by the nonlinear differential equations

$$
\begin{aligned}
\frac{\mathrm{d} c_{1}}{\mathrm{~d} t}= & k_{0 \rightarrow A_{1}}-k_{A_{1} \rightarrow 0} c_{1}-k_{A_{1}+A_{3} \rightarrow A_{4}} c_{1} c_{3} \\
& +k_{A_{4} \rightarrow A_{1}+A_{3}} c_{4}-k_{A_{1}+A_{4} \rightarrow A_{5}} c_{1} c_{4} \\
& +k_{A_{5} \rightarrow A_{1}+A_{4}} c_{5}, \\
\frac{\mathrm{d} c_{2}}{\mathrm{~d} t}= & -k_{A_{2} \rightarrow 0} c_{2}+k_{A_{4} \rightarrow A_{2}+A_{3}} c_{4} \\
& +k_{A_{5} \rightarrow A_{2}+A_{4}} c_{5}, \\
\frac{\mathrm{d} c_{3}}{\mathrm{~d} t}= & -k_{A_{1}+A_{3} \rightarrow A_{4}} c_{1} c_{3}+k_{A_{4} \rightarrow A_{1}+A_{3}} c_{4} \\
& +k_{A_{4} \rightarrow A_{2}+A_{3}} c_{4},
\end{aligned}
$$




$$
\begin{aligned}
\frac{\mathrm{d} c_{4}}{\mathrm{~d} t=} & k_{A_{1}+A_{3} \rightarrow A_{4}} c_{1} c_{3}-k_{A_{4} \rightarrow A_{1}+A_{3}} c_{4} \\
& -k_{A_{4} \rightarrow A_{2}+A_{3}} c_{4}-k_{A_{1}+A_{4} \rightarrow A_{5}} c_{1} c_{4} \\
& +k_{A_{5} \rightarrow A_{1}+A_{4}} c_{5}+k_{A_{5} \rightarrow A_{2}+A_{4}} c_{5}, \\
\frac{\mathrm{d} c_{5}}{\mathrm{~d} t=} & k_{A_{1}+A_{4} \rightarrow A_{5}} c_{1} c_{4}-k_{A_{5} \rightarrow A_{1}+A_{4}} c_{5} \\
& -k_{A_{5} \rightarrow A_{2}+A_{4}} c_{5} .
\end{aligned}
$$

(The rate constants $k_{A_{1} \rightarrow 0}$ and $k_{A_{2} \rightarrow 0}$ are both equal to the reciprocal of the reactor residence time, $1 / \theta$, and the rate constant $k_{0 \rightarrow A_{1}}$ equals $c_{1}^{f} / \theta$ with $c_{1}^{f}$ denoting the concentration for species $A_{1}$ in the feed stream.)

A spanning-subnetwork of network (11 b) is chosen

$$
\begin{aligned}
& A_{1} \rightarrow 0 \leftarrow A_{2}, \\
& A_{1}+A_{3} \rightarrow A_{4} \rightarrow A_{2}+A_{3}, \\
& A_{1}+A_{4} \rightarrow A_{5} \rightarrow A_{2}+A_{4} .
\end{aligned}
$$

By (3a), its two $(r-p-s=9-3-4)$ spanning-subnetwork vectors $d^{(1)}, d^{(2)}$ consist of the elements

$$
\begin{aligned}
& d_{A_{1} \rightarrow 0}^{(1)}=-1, \quad d_{A_{2} \rightarrow 0}^{(1)}=1, \\
& d_{A_{1}+A_{3} \rightarrow A_{4}}^{(1)}=1, \quad d_{A_{4} \rightarrow A_{2}+A_{3}}^{(1)}=1, \\
& d_{A_{1}+A_{4} \rightarrow A_{5}}^{(1)}=0, \quad d_{A_{5} \rightarrow A_{2}+A_{4}}^{(1)}=0, \\
& d_{A_{1} \rightarrow 0}^{(2)}=-1, \quad d_{A_{2} \rightarrow 0}^{(2)}=1, \\
& d_{A_{1}+A_{3} \rightarrow A_{4}}^{(2)}=0, \quad d_{A_{4} \rightarrow A_{2}+A_{3}}^{(2)}=0, \\
& d_{A_{1}+A_{4} \rightarrow A_{5}}^{(2)}=1, \quad d_{A_{5} \rightarrow A_{2}+A_{4}}^{(2)}=1 .
\end{aligned}
$$

According to the conditions (i) and (ii) of analysis, from (12) we have

$$
\begin{aligned}
& {\left[\xi_{1}\left(\phi_{1}\right)+\alpha_{1}\right](-1)+\left[\xi_{2}\left(\phi_{1}\right)+\alpha_{2}\right](-1)} \\
& \text { and }-\alpha_{1}-\alpha_{2} \text { are s.c.w. }-\phi_{1}, \\
& {\left[\xi_{1}\left(\phi_{1}+\phi_{3}\right)+\alpha_{1}\right](1)} \\
& \text { and }\left[\xi_{1}\left(\phi_{4}\right)+\alpha_{1}\right](1) \text { are s.c.w. } \phi_{4}-\phi_{1}-\phi_{3}, \\
& {\left[\xi_{2}\left(\phi_{1}+\phi_{4}\right)+\alpha_{2}\right](1)} \\
& \text { and }\left[\xi_{2}\left(\phi_{5}\right)+\alpha_{2}\right](1) \text { are s.c.w. } \phi_{5}-\phi_{1}-\phi_{4}, \\
& \xi_{1}(1)+\xi_{2}(1)>0, \\
& {\left[\xi_{1}\left(\phi_{2}\right)+\alpha_{1}\right](1)+\left[\xi_{2}\left(\phi_{2}\right)+\alpha_{2}\right](1)=0,} \\
& \xi_{1}(1)>0, \quad\left[\xi_{1}\left(\phi_{4}\right)+\alpha_{1}\right](1)=0, \\
& \xi_{2}(1)>0, \quad\left[\xi_{2}\left(\phi_{5}\right)+\alpha_{2}\right](1)=0 .
\end{aligned}
$$

The mass conservation condition for this example indicates that $\mathrm{d}\left(c_{3}+c_{4}+c_{5}\right) / \mathrm{d} t=0$. A vector $\gamma \in S_{t}$ if and only if $\gamma_{3}+\gamma_{4}+\gamma_{5}=0$. Thus a vector $\boldsymbol{\phi}=\left[\phi_{1}, \phi_{2}, \ldots, \phi_{5}\right] \in \mathfrak{R}^{5}$ is sign compatible with the stoichiometric subspace for

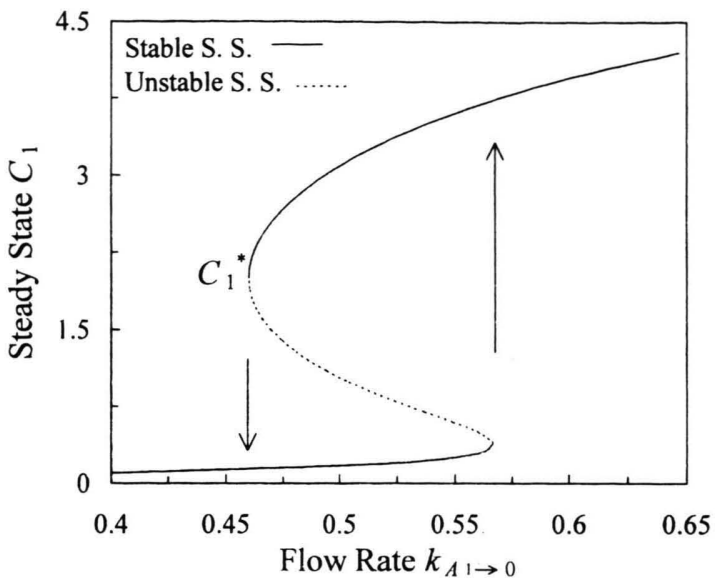

Fig. 1. The locus of steady state $c_{1}$ with the flow rate $k_{A_{1} \rightarrow 0}$ for network (15a).

network (11) if and only if the set $\left[\phi_{3}, \phi_{4}, \phi_{5}\right]$ contains both a positive and negative number, or else consists entirely of zeros. A sign compatible (nonzero) vector $\boldsymbol{\phi}$ and unknowns $\xi_{1}, \xi_{2}, \alpha_{1}, \alpha_{2}$ satisfying the inequality system (13) are found:

$\xi_{1}=1.45, \xi_{2}=0.67, \alpha_{1}=2.175, \alpha_{2}=-0.335$,

$\boldsymbol{\phi}=\left[\phi_{1}, \ldots, \phi_{5}\right] \approx[2,-0.8679,-3.5,-1.5,0.5]$.

According to the zero eigenvalue analysis, there exists a set of positive rate constants $\left\{k_{i \rightarrow j}: i \rightarrow j \in R\right\}$ such that its positive steady state $c^{*}$ has a zero eigenvalue with its eigenvector $\gamma$ in the stoichiometric subspace for network (11). By a choice of $\gamma=[4,-4,-0.5,-3,3.5]$, from (8) they are

$$
\begin{aligned}
& A_{1} \stackrel{0.46}{\stackrel{3.04}{\rightleftarrows}} 0 \stackrel{0.46}{\longleftarrow} A_{2}, \\
& A_{1}+A_{3} \underset{0.75}{\stackrel{10.325}{\rightleftarrows}} A_{4} \stackrel{0.725}{\longrightarrow} A_{2}+A_{3} \text {, } \\
& A_{1}+A_{4} \underset{0.2857}{\stackrel{0.6675}{\rightleftarrows}} A_{5} \stackrel{0.0957}{\longrightarrow} A_{2}+A_{4} \text {, } \\
& c^{*}=\left[c_{1}^{*}, c_{2}^{*}, \ldots, c_{5}^{*}\right] \approx[2,4.6087,0.1429,2,7]
\end{aligned}
$$

Equation (15) is used to simulate local steady states around $c^{*}$. The result is displayed in Figure 1. The steady state $c^{*}$ in Fig. 1 is a turning point with the values given in (15b). Perturbing a little the flow rate $k_{A_{1} \rightarrow 0}$, there exist two steady states near $c^{*}$. They become closer and closer and finally meet at $c^{*}$. There is a region having three steady states, one (middle) unstable and two (upper and lower) stable. The bistability occurring in the network (15a) is illustrated as hysteresis with variation of the flow rate. 


\section{Concluding Remarks}

A reaction network can admit multiple positive steady states if and only if there exists a positive steady state having a zero eigenvalue with its eigenvector in the stoichiometric subspace. The zero eigenvalue analysis provides a necessary and sufficient condition for the determination of such a steady state. Since both networks (1) and (11) have deficiency two, neither the deficiency zero theorem nor the deficiency one theorem (or algorithm) can be applied. Clarke [13] has shown that network (1), the Oregonator model, admits no multiple steady states by stoichiometric network analysis. Network (1) exhibits an unstable steady state [15]. If a network has only one steady state, an unstable steady state will probably come from a Hopf bifurcation [14]. This is the case for network (1). It is a wide spread belief [1] that any reasonable model of an oscillatory chemical reaction is expected to show bistability for another region of the parameter values. Apparently the Oregonator model gives an exceptional example. If stoichiometric network analysis is applied to study network (11), one should find an unstable steady state. However, an unstable steady state

[1] P. Erdi and J. Toth, Mathematical Models of Chemical Reactions, 1989.

[2] H. G. Othmer, Chem. Eng. Sci. 31, 993 (1976).

[3] D. Luss, Dynamics and Modeling of Reactive Systems, $131,1980$.

[4] D. Luss, Modeling of Chemical Reaction Systems, 305, 1981.

[5] P. Gray and S. K. Scott, Chem. Eng. Sci. 38, 29 (1983).

[6] P. Gray and S. K. Scott, J. Phys. Chem. 87, 1835 (1983).

[7] M. Feinberg, Chem. Eng. Sci. 42, 2229 (1987).

[8] F. J. M. Horn, Arch. Rational Mech. Anal. 49, 172 (1972).

[9] F. J. M. Horn and R. Jackson, Arch. Rational Mech. Anal. 47, 81 (1972). may go to a boundary (one or more species have zero concentrations), explosion (one or more species have infinite concentrations), another stable steady state, stable limit cycle or other chaotic attractors. This needs further numerical analysis. However, the zero eigenvalue analysis proposed in this work can directly answer the question of multiple steady states.

The zero eigenvalue analysis in this work studies two nearby steady states, while the method proposed in [17] studies those which may or may not be nearby. The conditions in zero eigenvalue analysis involve terms, $\boldsymbol{y}_{i} \cdot \boldsymbol{\phi}$, and are easier to analyze. The terms $\exp \left(\boldsymbol{y}_{i} \cdot \boldsymbol{\mu}\right)$, involved in the other method, are highly nonlinear and are more difficult to study. However, the vector $\boldsymbol{\mu}$ is related to two positive steady states $c^{*}$ and $c^{* *}$ by $\mu=\ln \left(c^{*} / c^{* *}\right)$, which may be measured in experiments. However, the vector $\phi$ defined in (8a) may not be measured directly.

\section{Acknowledgement}

The author thanks the National Science Council of R.O.C. (NSC 87-2214-E-239-001) for financial support of this work.

[10] M. Feinberg, Arch. Rational Mech. Anal. 49, 187 (1972).

[11] M. Feinberg, Chemical Reactor Theory: A Review, Chapt. 1, 1977.

[12] M. Feinberg, Chem. Eng. Sci. 43, 1 (1988).

[13] B. L. Clarke, Adv. Chem. Phys. 42, 1 (1980).

[14] K. D. Willamowski, Z. Naturforsch. 30 a, 989 (1978).

[15] R. J. Field and R. M. Noyes, J. Chem. Phys. 60, 1877 (1974).

[16] H. Y. Li, Ph.D. Dissertation, University of Rochester, NY 1992.

[17] H. Y. Li, Submission for publication.

[18] J. E. Bailey and D. F. Ollis Biochemical Engineering Fundamentals, Chapt. 9, 1986. 